\title{
Factores que inciden en la implementación de las TIC en los procesos de enseñanza-aprendizaje en $5^{\circ}$ de Primaria en Colombia
}

\section{Factors affecting the implementation of ICT in teaching and learning processes in the $5^{\text {th }}$ level of a Colombian primary school}

\author{
Sandra Rocío PARRA SARMIENTO ${ }^{1}$, Marcela Georgina GÓMEZ ZERMEÑO² y \\ María Manuela PINTOR CHÁ VEZ ${ }^{2}$ \\ ${ }^{1}$ Universidad Antonio Nariño (Colombia) y ${ }^{2}$ Tecnológico de Monterrey (México)
}

Recibido: Septiembre 2014

Aceptado: Octubre 2014

\section{Resumen}

El propósito de este trabajo es de identificar y analizar los factores que inciden en el uso de las TIC en los procesos de enseñanza-aprendizaje en el nivel 5to de primaria en una escuela colombiana y plantear una estrategia de mejoramiento en su implementación. Se utilizó un enfoque cualitativo y se recolectó la información mediante entrevistas, observaciones y revisión de documentos. Los participantes fueron estudiantes y docentes del 5to grado, así como personal directivo de una escuela pública de nivel primaria, quienes brindaron sus opiniones acerca del uso de las TIC en su centro escolar. Entre los factores que inciden en la aplicación de herramientas tecnológicas en el ámbito escolar, se identificó la capacitación, la disponibilidad de recursos y el apoyo institucional. Como resultado, se encontró que todos los participantes reconocen los beneficios de las TIC, especialmente los estudiantes, sin embargo se necesita reforzar la capacitación y el apoyo de autoridades superiores.

Palabas clave: tecnologías de la información y de la comunicación, práctica pedagógica, escuela primaria, innovación pedagógica.

\begin{abstract}
The purpose of this paper is to identify and analyze the factors affecting the use of ICT in the teaching-learning process at the 5th grade, elementary level in a Colombian school; consequently, it is essential to create a proposal in order to improve its use. A qualitative method was used and the information was collected through interviews, observations and documents review. The participants of this study were 5th grade students, teachers and school officials of a public primary school, who shared their opinions about the use of ICT in their educational center. Among the factors that influence in the implementation of technological tools there can be found: training, availability of resources and institutional support. As a result,
\end{abstract}


it was found that all the participants acknowledge the benefits of ICT, especially students. However, enhancement is needed in training and support from higher authorities.

Keywords: information and communication technologies, teaching practice, primary school, educational innovation.

En los últimos años muchos países, entre ellos Colombia, han realizado grandes esfuerzos por actualizar su sistema educativo, al incorporar diferentes tecnologías en el aula, tendientes a generar habilidades digitales en las personas involucradas en los procesos de enseñanza-aprendizaje, como son docentes y estudiantes. A pesar de todo, los resultados obtenidos no han sido significativos debido a múltiples factores que van desde los recursos económicos, las estrategias de implementación de programas, la capacitación de docentes y la falta de motivación hacia el uso de las tecnologías de información y comunicación (TIC).

Las TIC son fundamentales para dinamizar las actividades de aprendizaje en las aulas escolares. En un contexto educativo, las TIC pueden ayudar a desarrollar en las personas las competencias necesarias para un buen desempeño en el campo personal, social y laboral.

Conforme crece el ámbito de la educación, se vuelve necesario incorporar propuestas innovadoras que fomenten la calidad educativa. Se requiere conocer la realidad donde se desee implementar las TIC con el fin de identificar las características y competencias de sus usuarios para realizar una evaluación informada sobre las necesidades de ese contexto (Rivero, Gómez-Zermeño y Abrego, 2013).

Al identificar y analizar los factores que inciden en el uso de las TIC en los procesos de enseñanza-aprendizaje, el estudio aporta a la educación elementos valiosos para lograr la inclusión de estas en el aula, situación que hasta ahora ha sido esquiva para muchas instituciones educativas colombianas.

La necesidad de realizar acciones de mejoramiento continuo de los procesos educativos, y específicamente el de enseñanza-aprendizaje, hace que surjan cuestionamientos sobre cuáles son las estrategias viables que se pueden desarrollar e implementar con los estudiantes, buscando una mayor apropiación del conocimiento. En particular el desarrollo de esta investigación será en el contexto Colombiano, país que todavía se encuentra en situación de rezago al respecto de la incorporación de las TIC y la apropiación docente de estas (Ministerio de Educación Nacional de Colombia, 2002 - 2006).

Por lo cual, se planteó la siguiente pregunta de investigación: ¿Cuáles son los factores que inciden en la implementación de las tecnologías de información y la comunicación (TIC), en el quinto grado de primaria en el área de Ciencias Naturales? En conjunto, se busca responder a las preguntas: ¿Cuáles son los factores que permiten a docentes de la institución de estudio implementar las TIC en el desarrollo de sus materias?, ¿Cómo las políticas educativas fomentan el desarrollo e implementación de las TIC en la educación primaria? 
Como objetivo general de estudio, se buscó determinar los factores que inciden en el uso de las TIC y plantear una estrategia de mejoramiento que facilite el proceso de enseñanza-aprendizaje en el área de Ciencias Naturales, en los estudiantes de quinto grado de primaria, del colegio bajo estudio, ubicado en la ciudad de Cúcuta, Colombia.

De igual forma, se pretende identificar los factores que faciliten a docentes de Ciencias Naturales del quinto grado de primaria implementar las TIC en el desarrollo de su materia, precisar la forma en que las políticas educativas fomentan el desarrollo y uso de TIC e investigar aquellas herramientas tecnológicas que los estudiantes utilizan con mayor frecuencia para complementar sus estudios.

\section{Marco Teórico}

El impacto que han causado las TIC en las actividades de la vida humana, incluyendo el área de educación ha sido mayúsculo, tanto así que se podría hablar de la educación antes de las TIC y después de ellas, convirtiéndose en un punto de referencia obligado. En el desarrollo de esta temática, se encuentran los siguientes subtemas que están estrechamente relacionados: El rol docente, la capacitación del docente en TIC, en particular en el contexto colombiano donde se llevó a cabo la investigación, y la innovación educativa.

\section{El rol docente}

Los docentes realizan un rol fundamental en la formación integral de sus estudiantes, despertando la creatividad, criticidad, favoreciendo el entendimiento, la tolerancia, la justicia, la equidad, el respeto por el medio ambientes, entre otros.

La acción docente en el aula debe ser mediadora, debe buscar que la actividad en la escuela sea didáctica y se involucren las dimensiones comunicativas y sociales en los procesos de enseñanza-aprendizaje. El papel de la escuela es ilustrar a los actores que intervienen en el proceso, en el uso consciente y crítico de los aparatos que almacenan la información y el conocimiento, ya que el mundo cada vez se encuentra más avanzado en el sentido de la tecnología.

El ambiente virtual de aprendizaje permite un proceso de interacción mediado por diversos instrumentos tecnológicos, sin embargo, se hace necesario concebir y diseñar dichos espacios desde una estrategia pedagógica que permita alcanzar un nivel de conocimiento y de significación de lo que se presenta, es así como el "Ambiente Virtual de Aprendizaje es el conjunto de entornos de interacción, sincrónica y asincrónica, donde, con base en un programa curricular, se lleva a cabo el proceso enseñanza-aprendizaje, a través de un sistema de administración de aprendizaje" (López Rayón, Escalera, Ledesma, 2002, citados en Chan, 2004, p. 3).

El éxito en la aplicación de las TIC en los planes curriculares está condicionado a nuevos planteamientos que el docente realice de su trabajo diario, pues si se continúa con una mentalidad tradicional y rígida la implementación de esta alternativa pedagógica muy seguramente fracasará. Dentro de una de las propuestas y sus pautas, 
está que se incorpore en las instituciones de educación, la unidad curricular de las TIC, para realizar una formación profesional a la vanguardia del mundo actual.

Al respecto la UNESCO (2008, p. 7) escribe: "Las nuevas tecnologías (TIC) exigen que los docentes desempeñen nuevas funciones y también, requieren nuevas pedagogías y nuevos planteamientos en la formación docente”. Lograr la integración de las TIC en el aula dependerá de la capacidad de los maestros y las maestras para estructurar el ambiente de aprendizaje de forma no tradicional, fusionar las TIC con nuevas pedagogías.

Es por eso que la implementación de las TIC se convierte hoy en día en uno de los principales retos que se le presenta a los docentes. Está en los profesores y las profesoras aceptar y contribuir al mejoramiento de la calidad de sus estudiantes, o por el contrario, continuar desarrollando su trabajo haciendo caso omiso a la realidad que se vive en el mundo entero y permitiendo que la juventud a la cual se está ayudando a formar, pierda la posibilidad de construir conocimiento con base en herramientas tecnológicas.

Salas (2005), después de hacer una investigación bibliográfica en el año 2005 sobre las TIC, hace una síntesis y reflexiona sobre alguno de los principales hallazgos de la investigación realizada en Estados Unidos para determinar los principales factores de éxito o fracaso como herramienta para generar cambios, mejorar los procesos de enseñanza aprendizaje y cómo aprovechar y explotar el potencial de estas tecnologías en la educación general.

La investigación encontró que los avances en la incorporación de las TIC, en la educación son pocos significativos. Al desmejorarse la educación (perder calidad) en los niveles de primaria y secundaria, afloraron los repercusiones a nivel de la universidad. Un inadecuado proceso de planeación y la falta de participación de los usuarios en el diseño y estructuración podrían ser las mayores fallas. También se determinó que los principales obstáculos para que las TIC sean empleadas adecuadamente en la enseñanza son: falta de planificación, visión, incentivos, soporte técnico, dinero para cubrir gastos, apoyo insuficiente en la investigación, limitada e inadecuada infraestructura, limitado acceso a los equipos, desarrollo profesional insuficiente del profesorado (capacitación).

Las TIC tienen el potencial de elevar la capacidad comunicativa de los docentes y si asumimos que el acto educativo es en esencia un acto de comunicación, cualquier medio que contribuya a mejorarla y hacerla más efectiva irá, a su vez a contribuir al mejoramiento de la enseñanza y el aprendizaje (Salas, 2005).

Las TIC como herramientas didácticas no solo brindan beneficios al estudiante en cuanto a la manera de concebir su educación, sino que también son capaces de ofrecer grandes ventajas para los docentes al ayudar a captar el interés de sus estudiantes, ya que por medio de estas herramientas se abren las posibilidades de comunicación y por lo tanto fortalece la relación docente-estudiante encaminado al mejoramiento del trabajo escolar, es decir la educación, y todo lo que en ella interactúa, juega un papel importante puesto que es concebida como promotora de valores compartidos y responsable del desarrollo creativo y afectivo de los educandos (UNESCO, 2008). 
La construcción del aprendizaje en las aulas escolares es una responsabilidad compartida entre docentes y estudiantes y mediante la utilización de la tecnología se podría motivar al estudiante para el aprendizaje continuo en las asignaturas en las escuelas (Martín-Laborda, 2005).

Las TIC exigen que los docentes desempeñen nuevas funciones y también, requieren nuevas pedagogías y nuevos planteamientos en su formación. Lograr la integración de las TIC en el aula dependerá de la capacidad de los profesores y las profesoras para estructurar el ambiente de aprendizaje de forma no tradicional, fusionar las TIC con nuevas pedagogías y fomentar clases dinámicas en el plano social, estimulando la interacción cooperativa, el aprendizaje colaborativo y el trabajo en grupo (UNESCO, 2008).

Los cambios en la práctica docente suponen saber dónde y cuándo se deben o no utilizar las TIC para realizar actividades y presentaciones en el aula, tareas de gestión y adquisición de conocimientos (UNESCO, 2008).

En este proceso de cambio, los docentes innovadores son clave, en la medida en que hagan un mejor uso pedagógico de los recursos tecnológicos, harán de sus clases, momentos agradables, dinámicos y lograrán una educación de mejor calidad.

\section{Capacitación docente en TIC}

El desarrollo tecnológico del mundo actual exige que los ambientes de aprendizaje en las Instituciones educativas se integren con las TIC, esta incorporación educativa depende en gran medida del manejo que el docente haga de ellas en su quehacer diario. Si el docente es competente en el manejo de herramientas tecnológicas se habrá dado un gran paso en la implementación de TIC en los centros educativos. Dentro de su investigación, Vera (2003) pudo constatar que el docente no tiene en cuenta las herramientas tecnológicas, pero en caso contrario, los estudiantes sí las aprovechan para obtener buenos rendimientos, como en el caso de la Internet. Lo anterior permite destacar la necesidad de preparar al docente para aplicar las TIC en el proceso educativo.

Es claro que en este momento no todos los docentes pueden cambiar sus prácticas educativas así quisieran hacerlo, debido al desconocimiento que muchos de ellos tienen con respecto a las TIC, por eso es necesario implementar mecanismos de capacitación que permitan integrar a los docentes con estas.

La UNESCO (2008) plantea que el primer proceso, y el más complejo, que se debe desarrollar para que las instituciones educativas puedan ser eficientes y estén a la vanguardia en el uso de las TIC, es el de desarrollar nociones básicas de TIC. Este proceso consiste "en preparar estudiantes, ciudadanos y trabajadores, para que sean capaces de comprender las nuevas tecnologías y puedan así apoyar el desarrollo social y mejorar la productividad económica” (UNESCO, 2008, p. 8). Esta sería la base para empezar con el desarrollo de los programas encaminados a implementar las TIC. 
Cuando se diseñan los materiales didácticos, los profesores y las profesoras deben tener en cuenta no solo los objetivos que se pretende conseguir sino también las características del medio en que se van a utilizar, esto presupone un nuevo rol del docente y de los estudiantes (Olivar y Daza, 2007).

La generalización de las TIC en la educación por sí solas no garantiza el aprendizaje. Adicionalmente, existen algunos obstáculos que impiden el éxito de las TIC como (Márquez, 2006):

- Ritmo acelerado de evolución que no permite manejarlas eficazmente.

- Indefinición de los objetivos que se buscan.

- Contradicciones sobre la terminología.

- Puntos de vista divergentes respecto al adecuado empleo de las TIC en educación.

El desarrollo de una buena práctica docente depende en gran medida de la calidad de los recursos que se seleccionen y además que se utilicen adecuadamente, acordes con los objetivos propuestos (Olivar y Daza, 2007).

Frau y Torrent (2009) mencionan que los planteles educativos, en su afán de mantenerse actualizados tecnológicamente, dedican buena parte de sus recursos en infraestructura y equipos, sin embargo descuidan factores tan importantes como la formación de sus docentes y la revisión y ajuste de sus currículos. El docente está llamado a realizar un papel fundamental en la formación integral de sus estudiantes, su acción en el aula debe ser mediadora, orientadora de procesos que promuevan la creatividad, el entendimiento, la tolerancia, la equidad, la autorregulación, el aprendizaje significativo y el respeto por el medio ambiente, entre otros.

$\mathrm{Al}$ respecto, la UNESCO (2008) señala que las TIC exigen que los docentes desempeñen nuevas funciones y requieren nuevas pedagogías y planteamientos en la formación docente. Obtener la integración de las TIC en el aula dependerá de la capacidad de los docentes para estructurar el ambiente de aprendizaje de forma no tradicional y fusionar las TIC con pedagogías nuevas. Los roles del docente se pueden enmarcar dentro del campo organizacional, para estimular la participación, intelectual, para enfocar puntos primordiales, recapitular, evaluar, etc., y social para propiciar ambientes positivos de aprendizaje. Uno de los roles más importantes es el de moderar las discusiones que se generan.

Frente a la revolución tecnológica, los docentes deben replantear su enseñanza y aportar nuevas propuestas y recursos didácticos a su práctica educativa, acoger la tecnología digital y dejar de ser simple observadores en un proceso que no se detiene.

La capacitación de los docentes es esencial, ya que "los profesores se convierten siempre en los mediadores y agentes básicos de las innovaciones (siempre como aplicadores y con frecuencia como investigadores y planificadores de cambios)" (Zabalsa 2002, citado por Salas, 2005, p.14). 


\section{Apoyo estatal en la educación en Colombia}

El apoyo del Estado a través de sus políticas y programas es un factor que incide profundamente en la implementación de las TIC.

El Gobierno Nacional, consciente de la importancia que tienen las tecnologías de información y comunicación, creó el Ministerio de las Tecnologías de la Información y las Comunicaciones y el 30 de julio de 2009 sancionó la ley 1341 que se constituyó en el marco legal para el acceso y uso de las TIC, el desarrollo de las tecnológicas de información y comunicación y las convierte en políticas de Estado de cumplimiento obligatorio.

La UNESCO (2008) estableció normas relacionadas a las competencias en TIC para docentes, este documento ha servido como marco de referencia para establecer la normatividad actual sobre el uso de estas tecnologías. Amparadas en las normas anteriores, las TIC entraron a formar parte de los currículos en los planteles educativos.

Los Ministerios de Educación Nacional y de las TIC serán responsables de (Colombia ley de TIC 2009, p. 2) "fomentar el emprendimiento de las TIC desde los establecimientos educativos, poner en marcha un sistema de alfabetización digital y capacitar en TIC a los docentes de los diferentes niveles educativos”.

El Ministerio de Educación Nacional de Colombia (2002 - 2006), dice que la revolución educativa busca dar respuesta a las necesidades de cobertura y calidad que requiere el país para alcanzar mejores condiciones de desarrollo social y económico, y mejorar la calidad de vida de la población. Para cumplir este objetivo, el plan de desarrollo educativo ha definido cuatro políticas educativas básicas: ampliar la cobertura educativa, mejorar la calidad de la educación, capacitar a los docentes y mejorar la eficiencia del sector educativo.

- Política de ampliación de cobertura educativa. Esta política pretende ampliar la cobertura territorial y poblacional, tanto en el sector urbano como en el rural, buscando una mejor equidad en la distribución de oportunidades. La política incluye dos programas básicos: la reorganización que busca el mejoramiento de la gestión de las entidades, mejor utilización de recursos y optimización de la capacidad instalada y la asignación de recursos adicionales, para atender poblaciones marginadas del sistema educativa como: desplazados del conflicto colombiano, población indígena, niños con limitaciones, discapacidades y población de las áreas rurales. Hay dos factores que inciden negativamente en la ampliación de la cobertura: los desplazamientos forzados de familias y docentes del sector rural al sector urbano, y la explotación de niños en trabajos que los alejan de las aulas, a veces con la aquiescencia de sus progenitores que ven en ellos una forma de equilibrar sus presupuestos familiares.

- Política de mejoramiento de la calidad educativa. El mejoramiento de la calidad se constituye en el pilar fundamental del plan de desarrollo educativo. Los programas de incremento de cobertura deben ir acompañados de una política de calidad que movilice el sistema educativo en función del mejoramiento de los esquemas de aprendizaje y de la motivación de los niños por el acceso al conocimiento. El objetivo de las políticas de calidad es lograr que los estudiantes aprendan lo que 
necesitan aprender y lo sepan aplicar y aprovechar a lo largo de la vida. En la política de calidad el gobierno se propone poner en marcha un sistema de mejoramiento continuo, a partir de tres elementos: definición y difusión de estándares educativos, socialización de los resultados de un sistema de evaluación periódica y formulación de planes de mejoramiento desde las instituciones educativas. El programa "Colombia aprende" es un recurso que ayuda a esta política facilitando el acceso a contenidos de calidad y mostrando una manera distinta de concebir la pedagogía.

- Política de mejoramiento de la eficiencia del sector educativo. El sector educativo necesita replantear y modernizar sus estructuras institucionales, sus procedimientos administrativos y operativos, así como sus sistemas de incentivos y supervisión. Esta política está enfocada a la modernización de la administración y la gestión con tal propósito se implementaron cuatro programas orientados a asegurar la calidad de las inversiones, mejorar la productividad, la eficiencia y la trasparencia sectorial. El Ministerio de Educación Nacional (MEN) debe mejorar la calidad y oportunidad de sus programas de asistencia técnica y poner en marcha un sistema de información oportuno y confiable, que permita tomar decisiones, que facilite la planeación de la políticas, la asignación equitativa y eficiente de recursos, la oportuna evaluación y seguimiento de sus programas, debe generar incentivos a la eficiencia, socializar las experiencias más exitosas, promover la descentralización y la autonomía y facilitar la coordinación entre instituciones.

- Políticas de formación docente. El Ministerio de Educación Nacional como ente regulador y legislador de las políticas educativas y en cumplimiento de su responsabilidad constitucional, ha establecido como uno de sus actividades prioritarias la consolidación de la política nacional de formación de educadores. Esta política debe articular de manera coordinada a todos los actores del proceso formativo, tanto de los que inician como los docentes que ya están vinculados al servicio, esto fortalecerá la calidad del sistema educativo nacional.

\section{Innovación educativa}

La incorporación de las TIC en el aula genera procesos de innovación pedagógica que se expresan en una serie de cambios, como el rol del docente y del estudiante, cambios en los espacios físicos, cambios en la didáctica, en la manera de evaluar, en la utilización de recursos, que tanto el personal directivo, como docentes y estudiantes estén dispuestos a realizar y asumir las responsabilidades que se derivan de esta transformación.

El potencial de las tecnologías es muy grande y un docente innovador capaz de visualizar sus beneficios las puede convertir en un aliado en sus procesos de enseñanza-aprendizaje. La innovación no es una actividad puntual sino un proceso que debe ir acompañado de asesoramiento, reflexión, investigación, formación y evaluación (Cañal, 2002).

Se propone que este proceso innovador debe centrarse en el desarrollo de las competencias informacionales y digitales, enfocadas a preparar a los estudiantes como 
ciudadanos y ciudadanas autónomos, creativos y críticos. Se puede concluir que la presencia y utilización de las TIC en el aula, los cambios metodológicos en la enseñanza y la sola formulación curricular de metas educativas y difundidas en documentos oficiales no asegura la implementación de las TIC en las escuelas.

Las TIC e Internet, por sus características potenciales convierten el aula en una puerta abierta a enormes cantidades de información, que si se le da un uso adecuado en el aula, prestaría un enorme servicio a la educación. Salinas (2004) plantea cómo usarlas y recomienda que las instituciones deben ser flexibles y relacionarse estrechamente con su entorno para asegurar su vigencia, promover la integración de las tecnologías en los procesos de formación, el docente debe ejercer un liderazgo en los procesos de cambio y asumir su nuevo rol de guía, orientador y mediador.

La innovación pedagógica es la incorporación de novedades del momento. Sin embargo, estas novedades educativas no pueden ser arbitrarias o simplemente copiadas de otras experiencias, sino que deben ajustarse y responder a las necesidades específicas que se genera en el contexto educativo en donde se desenvuelve los docentes y sus estudiantes. Es en dicho contexto en donde el docente encuentra la motivación para realizar un cambio en su actuar, es decir asume una nueva percepción de sí mismo y se convierte en un gestor de recursos de aprendizaje. De esta manera, la innovación es utilizada para el uso y organización de recursos físicos y humanos con el fin de alcanzar los objetivos previamente definidos (Salinas, 2004).

La innovación se da como producto del análisis reflexivo que hace el docente durante sus prácticas pedagógicas. En este sentido, reflexionar significa tener consciencia sobre las acciones que se llevan a cabo durante el proceso de enseñanzaaprendizaje, conocer qué hacemos y cómo lo hacemos; para Schön (1998), "la reflexión es el motor o dinamizador del conocimiento. Gracias a la reflexión, el conocimiento supone experiencia transformada".

La innovación en la práctica pedagógica no se evidencia únicamente en la adquisición de nuevos conocimientos teóricos adquiridos por los docentes (Schön, 1998), sino que además debe estar sustentada en la ejecución de acciones concretas dentro del aula, en cuanto a actitudes, métodos didácticos utilizados, formas de evaluación y ejecución de planes de mejoramiento.

El proceso para promover docentes innovadores no solo depende de la formación y capacitación en técnicas y conceptos. Díaz-Barriga (2010), expone que cuando los docentes inician el proceso de innovación están sometidos a varias situaciones, la modificación de sus creencias y actitudes, transformar sus prácticas, implicaciones de carácter motivacional, concepciones didácticas, sus experiencias previas, entre otras.

La escuela debe producir los cambios necesarios para acercarse a la realidad del entorno en que está inmersa y mediante acciones innovadoras generar desarrollo institucional y comunitario. El propósito de la innovación es el mejoramiento constante de los proceso de enseñanza-aprendizaje, para ello, los docentes debe asumir la responsabilidad de identificar las situaciones que se presentan en el proceso educativo. 
Después de revisar la literatura para el marco teórico se destacan algunas coincidencias entre los autores referenciados.

- Todos coinciden en reconocer la importancia del docente en los procesos de cambio y en la implementación de las TIC.

- Reconoce que las TIC generan cambios y que estos modifican los roles de docentes y estudiantes.

- Valoran el uso de las TIC y su potencial en los procesos educativos.

- Analizan y destacan los beneficios de las TIC y son pocos los autores que le encuentran limitaciones y peligros si se usan adecuadamente.

\section{Metodología}

Para el desarrollo de esta investigación, se optó por un enfoque cualitativo. Los diseños basados en el enfoque cualitativo a menudo se denominan diseños emergentes, ya que emergen sobre la marcha. Esto quiere decir que el diseño puede cambiar según se va desarrollando la investigación, el investigador va tomando decisiones en función de lo que va descubriendo. El diseño basado en el enfoque cualitativo refleja el deseo que la investigación tenga como base de la realidad y los puntos de vista de los participantes (Salamanca y Martín-Crespo, 2007).

El centro escolar bajo estudio es una escuela en la ciudad de Cúcuta, departamento Norte de Santander, en la república de Colombia. Los participantes de la investigación fueron estudiantes de quinto grado de primaria, docentes y directivos de la institución educativa, durante los meses de enero-julio 2013.

Los sujetos de estudio fueron 16 estudiantes del quinto grado de educación primaria. Al seleccionarlos se tuvo en cuenta el principio de equidad de género y su voluntad de colaborar en el estudio. Por otro lado, tres docentes integraron la muestra, la titular del grado quinto, la encargada del área de ciencias naturales y el profesor de informática. Dos directivos se vincularán a la muestra, ellos expresaron su interés por la implementación de las TIC. De esta manera, la muestra se integró por un total de 21 personas.

Para esta investigación, se utilizaron tres instrumentos propios de la investigación cualitativa de tipo fenomenológico como son: la entrevista, la observación y la revisión de documentos. Para el análisis de la información obtenida se empleó la técnica de la categorización, los datos se registraron cuidadosamente y se redujeron a unidades llamadas categorías para facilitar el proceso de manejo y de interpretación.

Para la aplicación de los instrumentos fue necesario, contar con la autorización escrita de la rectora del plantel educativo, la aquiescencia de los docentes que participaron y el consentimiento de los padres y madres de familia para que sus hijos e hijas colaborara en la investigación, por ser menores de edad. Se explicaron los objetivos del trabajo y el manejo adecuado y responsable de la información captada. 
Se llevó a cabo la observación de una clase de la profesora encargada de impartir la asignatura de ciencias naturales en el quinto grado de primaria. La investigadora se comportó como un agente pasivo, no intervino en el desarrollo de la clase y solamente realizó las anotaciones de todos los aspectos relevantes para la investigación.

Para la aplicación de entrevistas a directivos y docentes, la entrevistadora llevaba una lista orientadora elaborada previamente. Al finalizar cada entrevista se empleó la técnica de verificación con el participante (Valenzuela y Flores, 2012), para asegurar la validez de la información. En la aplicación de la entrevista a los estudiantes, se les dio libertad de conformarlas libremente.

Para la revisión de documentos de los docentes, se analizaron las hojas de vida que utilizan en la institución de estudio, bajo el consentimiento de los docentes.

Para el análisis de los datos cualitativos, se aplicó el siguiente proceso (Valenzuela y Flores, 2012):

- Colección de datos.

- Preparación de datos para análisis

- Lectura de los datos para obtener un sentido general del material.

- Localización de segmentos del texto y codificación.

- Determinación y codificación de las categorías.

Los mismos autores (Valenzuela y Flores, 2012) señalan que el procedimiento de construcción de categorías es altamente inductivo; las categorías deberán cumplir los siguientes criterios:

- Deben responder a los propósitos del estudio, las categorías son las respuestas a las preguntas de investigación.

- Deben ser exhaustivas, deberán dar lugar a los datos que el investigador decida que son importantes y relevantes.

- Ser mutuamente excluyentes, una unidad de datos debe pertenecer a una y sola a una categoría.

- Ser congruentes, esto es, el mismo nivel de abstracción debe caracterizar a todas las categorías.

La manera principal de presentar y reportar una investigación cualitativa es en forma de una discusión narrativa (Valenzuela y Flores, 2012), es decir un resumen detallado de los principales hallazgos del análisis de datos, el cual se presenta en la siguiente sección.

\section{Resultados}

Se presenta un análisis de los resultados de la investigación con los datos más relevantes de una manera clara y ordenada, interpretando su articulación con la pregunta de investigación, ¿Cuáles son los factores que inciden en la implementación de las tecnologías de la información y la comunicación (TIC), en los procesos de enseñanza-aprendizaje, en el quinto grado de educación primaria en el área de ciencias naturales? 
Los docentes entrevistados expresaron que inicialmente sus clases eran magistrales, con muy poca participación de los estudiantes y con el apoyo de textos institucionales. Ante el caso anterior rondaba la incertidumbre si todos los docentes continuaban con sus clases magistrales y se procedió a observar el desarrollo de una clase con otro participante, quien utilizó los equipos disponibles, motivó a los estudiantes a través de preguntas, expuso el objetivo de aprendizaje, presentó el video y realizó una actividad colaborativa.

Los participantes están de acuerdo en que las TIC facilitan el aprendizaje y el logro de objetivos, promueven la interacción, afianzan conocimientos y le imprimen a las clases mayor motivación y dinamismo. Respecto a los equipos, afirman que son de buena calidad y que disponen de una infra-estructura física adecuada, pero son insuficientes para el número de estudiantes. Se consideran capacitados para manejar las TIC, con excepción de un docente.

El personal directivo manifestó que el colegio cumple con la infraestructura y equipos requeridos y considera que es una necesidad urgente de la educación actual.

En cuanto a los estudiantes, se observó que tienen claridad sobre las TIC y han usado las herramientas disponibles en el colegio. En lo más sobresaliente se notó que han tenido acceso a otros elementos tecnológicos en sus hogares. Han detectado algunas deficiencias en los equipos de cómputo en su centro escolar, como virus, baja velocidad en el internet, daños en accesorios y expresan la necesidad de mejorar el mantenimiento de accesorios y equipos.

La mayoría de los estudiantes están de acuerdo en proponer otros elementos tecnológicos que les gustaría utilizar en el colegio como: portátiles, cámaras, tabletas, iPad, celulares, etc. Coinciden con el grupo de docentes en que los equipos son insuficientes y dicen: "no quiero compartir mi computador con los demás".

De acuerdo con el proceso de análisis de datos planeado en la metodología, una vez obtenida la información a partir de los instrumentos se procedió a realizar las respectivas transcripciones con el fin de organizar los datos proporcionados. Por lo tanto, se elaboran tablas comparativas de las respuestas de los docentes durante la entrevista, las respuestas del personal directivo y de las respuestas de los estudiantes. Después de analizar detalladamente dichas tablas, se identificaron las incidencias para luego clasificarlas y conformar las categorías. 


\begin{tabular}{ll}
\hline Categoría & Incidencias \\
\hline $\begin{array}{l}\text { Factores que limitan el uso de las } \\
\text { (TIC) en el aula }\end{array}$ & $\begin{array}{l}\text { Clase Magistral } \\
\text { Apoyo en textos guía } \\
\text { Docentes no innovadores } \\
\text { Estudiantes pasivos }\end{array}$ \\
\hline $\begin{array}{l}\text { Factores que determinan la } \\
\text { influencia de las políticas } \\
\text { educativas en el uso de las (TIC) }\end{array}$ & $\begin{array}{l}\text { Normas UNESCO } \\
\text { Legislación Colombiana }\end{array}$ \\
$\begin{array}{ll}\text { Herramientas de las (TIC) que los } \\
\text { niños están dispuestos a utilizar } \\
\text { para complementar sus estudios }\end{array}$ & $\begin{array}{l}\text { Cámaras digitales } \\
\text { Celulares } \\
\text { Grabadoras }\end{array}$ \\
\hline $\begin{array}{l}\text { Factores externos que inciden en } \\
\text { la aplicación de las (TIC) }\end{array}$ & $\begin{array}{l}\text { Dabletas } \\
\text { Caponibilidad de recursos } \\
\text { Tabla 1. Factores de incidencia en la implementación de las TIC }\end{array}$
\end{tabular}

A pesar de disponer de políticas y programas estatales bien estructurados sobre el acceso y uso de las TIC, sin desconocer que se ha hecho mucho, los resultados no han sido los esperados, quizá ha existido negligencia en los niveles regionales y locales, que no han brindado un apoyo suficiente, ni han realizado seguimientos y controles estrictos que permitan medir la eficiencia y la efectividad de los programas para hacer los ajustes pertinentes. La política de mejoramiento de la eficiencia del sector educativo no se ha logrado implementarlo. Las Normas UNESCO relacionadas a las competencias TIC para docentes y los estándares educativos del Ministerio de Educación Nacional (MEN) han sido fundamentales en el diseño de políticas educativas.

Estudiantes y docentes manifestaron que los equipos son insuficientes frente a la demanda, además, presentan problemas de mantenimiento que deben ser resueltos.

Los niños y las niñas, a pesar de su corta edad, tienen claridad sobre las TIC y han usado los equipos disponibles en el colegio, en forma limitada. Reconocen la importancia y las ventajas que les ofrece la tecnología para: "investigar”, "hacer tareas", "hablar con amigos".

Al revisar las hojas de vida de los docentes, se ve claramente que la capacitación influye notoriamente en su desempeño. Existe un respaldo pleno del personal directivo del colegio para implementar las TIC y reconocen que "es una necesidad urgente de la educación actual y están dispuestos asumir el reto".

El personal directivo debe facilitar recursos humanos y financieros que favorezcan los procesos de capacitación docente y propicien el intercambio de experiencias en el uso de las TIC y crear incentivos para que los docentes abandonen sus clases tradicionales y se comprometan con el uso de las TIC. 
Los maestros y las maestras deben comenzar a gestionar y participar en procesos de formación y capacitación, tanto internos como externos, para contribuir a que su actitud cambie, sus temores disminuyan, su concepto en relación con las TIC se transforme y reconozcan las ventajas que estas herramientas tienen para estudiantes y para docentes.

Los docentes en ejercicio deben buscar una formación continua, adaptar la práctica investigativa y convertir su aula en un lugar de interacción entre docente-estudiante, que les permita la construcción de conocimientos significativos, y sirva para comprender y transformar su propia realidad. Los docentes reconocieron que inicialmente sus clases eran magistrales, apoyados en textos guías y poca participación de los estudiantes. Solo una de las profesoras expresó que ella había nacido con las TIC y no tenía ningún problema en aceptarlas y aplicarlas.

Algunos docentes continúan con sus clases magistrales y aducen las siguientes razones: "Me siento más segura haciéndolo así”, "Me quita mucho tiempo preparando las clases" y "Temo que se dañen los aparatos y tenga que pagarlos".

Los que utilizan las TIC expresaron: "Me gusta el trabajo que realizo", "He encontrado en las TIC el mejor aliado para mis clases, si todos los docentes supieran lo que facilita la enseñanza-aprendizaje trabajarían con ellas", "Siempre estoy pendiente de las novedades en educación”.

Los docentes con mayor tiempo de servicio, a pesar a su deseo y esfuerzo realizado por actualizarse, acusan carencias en el uso de las TIC desde el punto de vista conceptual y de aplicabilidad. La experiencia laboral que debería ser un factor favorable se torna en algunos casos negativa cuando los docentes se arraigan a sus clases tradicionales y se resisten al cambio.

Hay muchos docentes que tienen dificultades en el uso de las TIC y el manejo de los equipos, pero quienes sí las manejan manifestaron su interés en ayudar a sus compañeros y proponen que se diseñe un plan de mejoramiento y ampliación de cobertura.

La falta de motivación (intrínseca y extrínseca) y la falta de capacitación, tanto en TIC como en el manejo de equipos, son factores que obstaculizan la implementación de las TIC.

En lo que todos los docentes participantes están de acuerdo, es en que las TIC facilitan el aprendizaje y el logro de los objetivos, afianzan los temas, motivan, dinamizan las clases y favorecen la interacción estudiante-docente y estudianteestudiante.

Se puede decir que dentro de los factores analizados los que mayor inciden en la aplicación de las TIC son: la capacitación, la disponibilidad de recursos, el apoyo institucional y el cumplimiento de las políticas educativas establecidas. 


\section{Conclusiones}

Esta investigación estuvo enfocada en un contexto específico, en un solo salón de clases del $5^{\circ}$ grado de una escuela primaria colombiana; las conclusiones alcanzadas tienen un alcance limitado. Sin embargo, vale la pena señalarlas ya que existen todavía muchas escuelas primarias en América Latina que presentan características similares a las presentadas en este estudio, y el trabajo realizado podría ser una contribución en el tema, ya que ante los avances de la tecnología y el aval que las generaciones jóvenes le otorgan a las TIC, los docentes no tienen otra alternativa que la de prepararse para orientar adecuadamente su uso en los procesos de enseñanza-aprendizaje. A pesar de los esfuerzos realizados por el personal docente para asimilar los cambios que le generan el uso de las TIC en sus clases, presenta carencias, por lo que se necesita reforzar urgentemente el área de la capacitación.

La incorporación de las TIC en los currículos y las experiencias de los docentes que las están aplicando son factores positivos. No obstante, se requiere del apoyo de autoridades superiores en este sector, como son el Ministerio de Educación Nacional y la Secretaría Departamental de Educación ejercieran a cabalidad su función orientadora de vigilancia y evaluación.

El insuficiente nivel de competencia y la vergüenza que produce en los docentes el no saber manejar un recurso tecnológico, los incita a continuar con sus clases tradicionales, que les dan mayor seguridad y confianza, pero, generan resistencia al cambio y rechazo a diseñar actividades que involucre el uso de la herramientas TIC.

Aprovechando el pleno respaldo de los directivos, la propuesta de mejoramiento para implementación de las TIC en una escuela primaria colombiana, planteada en este documento, debe plasmarse en un proyecto institucional.

\section{Propuesta de mejoramiento}

Respecto al planteamiento de la propuesta de mejoramiento contemplada en el trabajo, se elaboró una en forma resumida, teniendo en cuenta las debilidades encontradas, que incluye los siguientes aspectos: capacitación de los recursos humanos, mejoramiento de los recursos físicos e implementación de acciones para promover el uso de las TIC.

Dentro de la capacitación de los recursos humanos estarían incluidos: a) Formación de docentes en TIC (utilizando las TIC en su formación), b) Capacitación en aspectos éticos y legales relacionados con las TIC, y c) Adiestramiento en el uso de equipos y programas.

En relación al mejoramiento de los recursos físicos se encuentra: La adquisición de equipos y un mantenimiento técnico adecuado y oportuno que puede ser preventivo y correctivo, en cuanto a programas informáticos y herramientas estaría adquirir materiales actualizados y respecto a redes y medios que sean ágiles, eficientes $\mathrm{y}$ actualizados. 
En cuanto a la implementación de acciones para promover el uso de las TIC estarían: la conformación de una red de usuarios de herramientas tecnológicas, establecimiento de estímulos para docentes innovadores, sensibilizar a directivos, padres y madres de familia, docentes y estudiantes sobre TIC, acceso a revistas arbitradas de avances tecnológicos, apoyos por parte de organismos estatales y el intercambio de experiencias sobre TIC con otras instituciones, ya sea de manera virtual o presencial. Esta propuesta tendría una duración de un año, con evaluaciones trimestrales y tendría que contar con el pleno respaldo de los directivos y el apoyo permanente del coordinador de informática.

\section{Referencias bibliográficas}

CAÑAL, P. (2002). La innovación educativa. Madrid, España: Akal.

CHAN, M. (2004). Tendencias en el diseño educativo para entornos de aprendizajes digitales. Revista digital universitaria 5(10), 1-26. Recuperado de: http://www.revista.unam.mx/vol.5/num10/art68/nov_art68.pdf

LEY DE TIC de agosto 17 de 2009. Artículos 20 y 67 de la constitución nacional del Estado (Colombia). Recuperado el 30 de enero de 2013 de: http://edutecno.org/2009/08/colombia-ley-de-tic-2009/

DÍAZ-BARRIGA, F. (2010). Los profesores ante las innovaciones curriculares. Revista Iberoamericana de Educación Superior, 1(1). Recuperado de: http://ries.universia.net

FRAU, D. Y TORRENT, J (2009) Políticas de educación en medio: hacia una propuesta global. Revista científica iberoamericana de comunicación y educación, 32(16), 10-14. Recuperado de: http://www.revistacomunicar.com/pdf/comunicar32.pdf

MÁRQUEZ, P. (2006). Taller de comunicación NTIC. Universidad de Barcelona, España. Recuperado de http://www.ubv.lmi/es

MARTÍN-LABORDA, R. (2005). Las nuevas tecnologías en la educación. Madrid, España: Fundación AUNA.

MINISTERIO DE EDUCACIÓN NACIONAL DE COLOMBIA. (2002 - 2006), Políticas educativas plan sectorial. Recuperado el 12 de marzo de 2013 de: http://www.mineducacion.gov.co/1621/propertyvalue-33708.html

OLIVAR, A. Y DAZA, A. (2007). Las tecnologías de la Información y Comunicación (TIC) y su impacto en la educación del siglo XXI. Revista Negotium, 3(7), 21-46. Recuperado de: http://www.revistanegotium.org.ve/7/Art2.pdf

RIVERO, I., GÓMEZ-ZERMEÑO, M.G. Y ABREGO, R. (2013). Tecnologías educativas y estrategias didácticas: criterios de selección. Revista electrónica Educación y Tecnología, (3), 190-206. Recuperado de http://revistas.umce.cl/edytec/article/view/134/141 
SALAMANCA A. B Y MARTÍN-CRESPO C. (2007). El diseño de la investigación cualitativa. Nure investigación, 26. Recuperado de: http://www.nureinvestigacion.es/FICHEROS_ADMINISTRADOR/F_METODOL OGICA/FMetodologica_26.pdf.

SALAS, F. (2005). Hallazgos de la investigación sobre la inserción de las Tecnología de la información y la comunicación (TIC) en la enseñanza: la experiencia de los últimos diez años en los Estados Unidos. Revista Educación, 29(2), 53-66.

SALINAS, J. (2004). Innovación Docente y Uso de las TIC's en la Enseñanza Universitaria. Revista de Universidad y Sociedad del Conocimiento, 1(1). Recuperado de: http://ries.universia.net

SCHÖN, D. A. (1998). El profesional reflexivo. Como piensan los profesionales cuando actúan. Barcelona: Paidós.

UNESCO (2008). Estándares de competencia en TIC para docentes. París: Unesco.

VALENZUELA, J.R., Y FLORES FAHARA, M. (2012). Fundamentos de investigación educativa. México: Editorial Digital del Tecnológico de Monterrey.

VERA, Y. (2003). El uso de la Internet como herramienta educativa y su relación en el rendimiento cualitativo de los alumnos del sexto grado de Educación Básica. Trabajo de Grado, Universidad Rafael Belloso Chacín. Maracaibo, Venezuela.

\section{Correspondencia con los autores}

\section{Sandra Rocío PARRA SARMIENTO}

Universidad Antonio Nariño

Avenida 4 \# 15-88 La Playa

Cúcuta, Norte De Santander, Colombia.

e-mail: saropasa@gmail.com

\section{Marcela Georgina GÓMEZ ZERMEÑO}

Escuela Nacional De Humanidades y Ciencias Sociales, Tecnológico de Monterrey

Avenida Eugenio Garza Sada 2501 Sur, Tecnológico

64849 Monterrey, N.L., México.

e-mail: marcela.gomez@itesm.mx

María Manuela PINTOR CHÁVEZ

Vicerrectoría de Programas en Línea, Tecnológico de Monterrey

Avenida Eugenio Garza Sada 2501 Sur, Tecnológico

64849 Monterrey, N.L., México.

e-mail: maria.pintor@tecvirtual.mx 